“ (C) 2018 IEEE. Personal use of this material is permitted. Permission from IEEE must be obtained for all other uses, in any current or future media, including

reprinting/republishing this material for advertising or promotional purposes, creating new collective works, for resale or redistribution to servers or lists, or reuse of any copyrighted component of this work in other works." 


\title{
Robust Digital Current Control Based on Adaptive Disturbance Estimation for PMSM Drives with Low Pulse ratio
}

\author{
Haitao Yang ${ }^{1,2}$,Yongchang Zhang ${ }^{2}$, Jiejunyi Liang ${ }^{1}$, Nong Zhang ${ }^{1}$ and Paul Walker ${ }^{1}$ \\ ${ }^{1}$ Faculty of Engineering and Information Technology, University of Technology Sydney, Sydney, Australia \\ ${ }^{2}$ Inverter Technologies Engineering Research Center of Beijing, North China University of Technology, Beijing, China \\ zyc@ncut.edu.cn
}

\begin{abstract}
For high-power or high-speed motor drives, the low switching frequency to fundamental frequency ratio leads to poor bandwidth or even instability of the current control loop if the controller is not properly designed. In this paper, a digital predictive current controller is constructed based on an exactly discretized model to overcome this issue. Then, a method of online disturbance adaptation is proposed to compensate for the side impact of motor parameter mismatches on the tracking performance. Additionally, online inductance adaptation is further incorporated to improve transient performance. Compared with the prior complex-vector proportional-integral controller, the proposed current controller presents faster dynamic responses and better parameter robustness. Simulation and experimental tests on a permanent magnet synchronous motor drive confirm the effectiveness of the proposed control schemes.
\end{abstract}

Index Terms-Digital current control, disturbance estimation, PMSM, low pulse ratio.

\section{INTRODUCTION}

Permanent magnet synchronous motor (PMSM) drives have been widely used in the applications of traction, lathe, renewable energy, etc. owing to their excellent efficiency, satisfactory controllability, and good power density [1]-[4]. For inverterfed PMSM, the operation under low switching frequency to fundamental frequency ratio may occur for high power and high speed applications [5]. In the first case, switching frequency is usually restrained at a low value to reduce switching losses, while in the second case the rated speed of the motor is designed at a high value, e.g. to reduce motor volume [5], [6]. In both cases, the discretization effect cannot be ignored and must be carefully considered. Otherwise, deteriorated control performance or even instability could be observed [7], [8]. Hence, the conventional method, i.e., designing the current controller in the continuous-time domain with subsequent discretization for digital implementation can not guarantee the desired performance.

To deal with the increased cross-coupling caused by relatively low sampling-rate, a first-order delay model is used in the design of a complex-coefficient current controller [9]. It is

This work was supported in part by the Australian Research Council under grant DP150102751, and in part by the National Natural Science Foundation of China under Grant 51577003. shown that the transient performance is significantly improved if the signal delay is explicitly considered. To address the issue of instability at high speed operation, a voltage compensation strategy is proposed in [7]. Both controllers in [7] and [9] are designed in the continuous-time domain and thus a discretization method is required for digital implementation. Without proper discretization, the controller performance may be degraded in practical implementation. For better performance, it is suggested to design controllers directly in the discrete-time domain [8], [10]. As the discrete nature of the digital implementation, such as sampling delay and zero-orderhold $(\mathrm{ZOH})$, can be explicitly considered in the design stage, the bandwidth and improved coupling performance may be obtained by using discrete-time controller.

To design discrete-time current controller, it is required to develop the discrete-time system model first. In [8], [10], two different discrete-time models are derived in the rotor-flux oriented synchronous-reference-frame. Then, the pole/zero cancellation principle is applied to develop the complex-coefficient current controller. Though the parameter robustness is improved when compared with the conventional proportional-integral controller, the desired performance depends on exact pole/zero cancellation. If motor parameter deviates from its nominal value, the control performance deteriorates. As in practical applications, the stator inductance and resistance may change due to saturation and temperature variation. Thus, it is essential to ensure the controller can handle parameter mismatches so that the desired performance can always be guaranteed.

To overcome the aforementioned issues, a digital predictive current controller (DPCC) is developed based on the exactly discretized PMSM model in this paper. Simulation and experimental results show that DPCC has fast dynamic responses but tracking error and instability may occur if motor parameters deviate from their nominal values. Hence, online disturbance estimation and inductance correction methods are further developed in the discrete-time domain to address performance deterioration resulted from the mismatched motor parameters. By combining the adaptive disturbance estimation, the proposed DPCC can achieve stable operation, fast dynamic 
response, and good robustness against parameter variation with very low pulse ratio.

\section{Mathematical Model of PMSM}

\section{A. Continuous-time PMSM Model}

The mathematical model of PMSM can be expressed in the form of complex vectors in the $\alpha-\beta$ stationary frame as:

$$
L \frac{d \boldsymbol{i}_{s}^{\alpha \beta}(t)}{d t}=\boldsymbol{u}_{s}^{\alpha \beta}(t)-R_{s} \boldsymbol{i}_{s}^{\alpha \beta}(t)-j \omega_{r}(t) \cdot \boldsymbol{\psi}_{r}^{\alpha \beta}(t) .
$$

where $\boldsymbol{u}_{s}, \boldsymbol{i}_{s}, R_{s}, L, \omega_{r}$ and $\psi_{r}$ are stator voltage vector, stator current vector, stator resistance, inductance, electrical rotor speed and permanent magnet flux vector respectively.

The following Park transformation (2)

$$
\boldsymbol{x}^{d q}=\boldsymbol{x}^{\alpha \beta} e^{-j \theta_{r}(t)}
$$

is applied to derive the dynamic model in the rotor-flux oriented synchronous reference frame as

$L \frac{d \boldsymbol{i}_{s}^{d q}(t)}{d t}=\boldsymbol{u}_{s}^{d q}(t)-R_{s} \boldsymbol{i}_{s}^{d q}(t)-j \omega_{r}(t) L \boldsymbol{i}_{s}^{d q}(t)-j \omega_{r}(t) \cdot \boldsymbol{\psi}_{r}^{d q}(t)$

where $\theta_{r}$ is rotor angle. Taking the back electromotive force (EMF) as the disturbance, the transfer function can be obtained as

$$
G^{d q}(s)=\frac{\boldsymbol{I}^{d q}(s)}{\boldsymbol{U}^{d q}(s)}=\frac{1}{R_{s}+s L+j \omega_{r} L}
$$

As seen in (4), there is a complex coefficient appears in the denominator, indicating the cross-coupling between $d$-axis and $q$-axis current [10].

\section{B. Discrete-time PMSM Model}

Assuming a constant speed during each sampling period, the model (1) can be described as

$$
\begin{array}{r}
\boldsymbol{i}_{s}^{\alpha \beta}(k+1)=\frac{1}{L} \int_{0}^{T_{s c}} e^{-\frac{R_{s}}{L}\left(t-T_{s c}\right)}\left(\boldsymbol{u}_{s}^{\alpha \beta}(t)-j \omega_{r} \cdot \boldsymbol{\psi}_{r}^{\alpha \beta}(t)\right) d t \\
+e^{-\frac{R_{s}}{L} T_{s c}} \boldsymbol{i}_{s}^{\alpha \beta}(k)
\end{array}
$$

For a inverter-fed PMSM, the voltage $\boldsymbol{u}_{s}^{\alpha \beta}$ is generated by the PWM, and it can be assumed as a constant during the sampling period, i.e.,

$$
\boldsymbol{u}_{s}^{\alpha \beta}(t)=\boldsymbol{u}_{s}^{\alpha \beta}(k)
$$

If only the fundamental component is considered, rotor flux vector can be expressed as

$$
\boldsymbol{\psi}_{r}^{\alpha \beta}(t)=\boldsymbol{\psi}_{r}^{\alpha \beta}(k) e^{j \omega_{r} t}
$$

Substituting (6) and (7) into (5) yields [11]

$$
\begin{array}{r}
\boldsymbol{i}_{s}^{\alpha \beta}(k+1)=e^{-T_{s c} / \tau} \boldsymbol{i}_{s}^{\alpha \beta}(k)+\frac{1-e^{-T_{s c} / \tau}}{R_{s}} \boldsymbol{u}_{s}^{\alpha \beta}(k) \\
-\frac{e^{j \omega_{r} T_{s c}-e^{-T_{s c} / \tau}}}{R_{s}+j \omega_{r} L} j \omega_{r} \boldsymbol{\psi}_{r}^{\alpha \beta}(k)
\end{array}
$$

where $\tau=\frac{L}{R}$ and $T_{s c}$ is the sampling period.

\section{Principle of the Proposed DPCC}

\section{A. Disturbance Estimation}

According to (8), the reference voltage that force the stator current to arrive its reference at the next sampling period, can be computed as

$$
\begin{array}{r}
\boldsymbol{u}_{s 1}^{r e f}(k)=\frac{\hat{R}_{s}}{1-e^{-T_{s c} / \hat{\tau}}}\left(\boldsymbol{i}_{s}^{r e f}(k+1)-e^{-T_{s c} / \hat{\tau}} \boldsymbol{i}_{s}^{\alpha \beta}(k)\right. \\
\left.+\frac{e^{j \omega_{r} T_{s c}-} e^{-T_{s c} / \hat{\tau}}}{\hat{R}_{s}+j \omega_{r} \hat{L}} j \omega_{r} \boldsymbol{\psi}_{r}^{\alpha \beta}(k)\right)
\end{array}
$$

where the hat ${ }^{\wedge}$ denotes the estimated variable. As (9) is directly calculated based on the system model, the calculated voltage would not achieve the desired tracking performance in practical application due to imperfect prior-knowledge of motor parameters, unknown disturbances, etc.

Using the estimated stator resistance $\hat{R}_{s}$ and stator inductance $\hat{L}$, the system model (1) can be rewritten as

$\hat{L} \frac{d \boldsymbol{i}_{s}^{\alpha \beta}(t)}{d t}=\boldsymbol{u}_{s}^{\alpha \beta}(t)-\hat{R}_{s} \boldsymbol{i}_{s}^{\alpha \beta}(t)-j \omega_{r}(t) \cdot \hat{\boldsymbol{\psi}}_{r}^{\alpha \beta}(t)-\boldsymbol{u}_{d}^{\alpha \beta}(t)$

where

$$
\boldsymbol{u}_{d}^{\alpha \beta}=-\Delta L \cdot d \boldsymbol{i}_{s}^{\alpha \beta}(t) / d t-\Delta R \boldsymbol{i}_{s}^{\alpha \beta}(t)-j \omega_{r}(t) \cdot \Delta \psi e^{j \theta_{r}(t)}
$$

is the disturbance voltage compensating for the inconsistency between (1) and (10); $\Delta L=L-\hat{L}, \Delta R=R_{s}-\hat{R}_{s}$ and $\Delta \psi=\psi_{r}-\hat{\psi}_{r}$ are estimation errors of inductance, resistance and rotor flux magnitude respectively. Similar to the derivation of (8), the following equation can be obtained

$$
\begin{array}{r}
\boldsymbol{i}_{s}^{\alpha \beta}(k+1)=e^{-T_{s c} / \hat{\tau}} \boldsymbol{i}_{s}^{\alpha \beta}(k)+\frac{1-e^{-T_{s c} / \hat{\tau}}}{\hat{R}_{s}} \boldsymbol{u}_{s}^{\alpha \beta}(k) \\
-\frac{e^{j \omega_{r} T_{s c}-} e^{-T_{s c} / \hat{\tau}}}{\hat{R}_{s}+j \omega_{r} \hat{L}}\left(j \omega_{r} \boldsymbol{\psi}_{r}^{\alpha \beta}(k)+\boldsymbol{u}_{d}^{\alpha \beta}(k)\right)
\end{array}
$$

It should be noted that only fundamental component of $\boldsymbol{u}_{d}^{\alpha \beta}$ is considered in (12). The dynamics of $\boldsymbol{u}_{d}^{\alpha \beta}$ can thus be expressed as

$$
\boldsymbol{u}_{d}^{\alpha \beta}(k+1)=e^{j \omega_{r} T_{s c}} \boldsymbol{u}_{d}^{\alpha \beta}(k)
$$

According to (12), the voltage forcing the stator current to reach its reference can be calculated as

$$
\begin{array}{r}
\boldsymbol{u}_{s 2}^{r e f}(k)=\frac{\hat{R}_{s}}{1-e^{-T_{s c} / \hat{\tau}}}\left(\boldsymbol{i}_{s}^{r e f}(k+1)-e^{-T_{s c} / \hat{\tau}} \boldsymbol{i}_{s}^{\alpha \beta}(k)+\right. \\
\left.+\frac{e^{j \omega_{r} T_{s c}-} e^{-T_{s c} / \hat{\tau}}}{\hat{R}_{s}+j \omega_{r} \hat{L}}\left[j \omega_{r} \boldsymbol{\psi}_{r}^{\alpha \beta}(k)+\boldsymbol{u}_{d}^{\alpha \beta}(k)\right]\right)
\end{array}
$$

Comparing (9) and (14), it can be seen that the additional disturbance voltage $\boldsymbol{u}_{d}^{\alpha \beta}$ is required to achieve the desired control target when the estimated motor parameters are used in the control algorithm. However, since the actual motor parameters is unknown, it is difficult to obtain its value directly. In the following text, an adaptive disturbance estimation would be developed. 


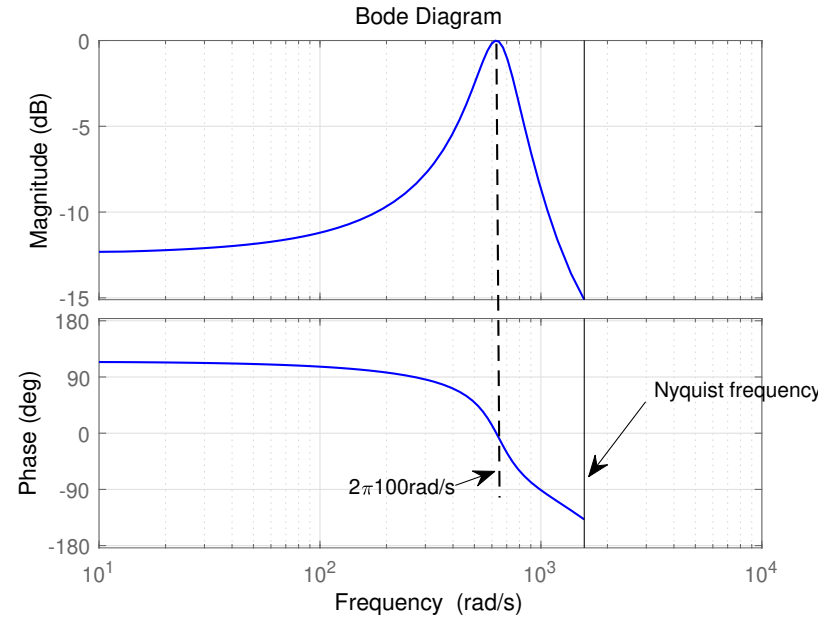

Fig. 1. Bode diagram of $F(z)$ at $100 \mathrm{~Hz}$ with $h=0.25$.

According to (12), the following equation can be derived

$$
\begin{gathered}
\boldsymbol{i}_{s}^{r e f}(k)=e^{-T_{s c} / \hat{\tau}} \boldsymbol{i}_{s}^{\alpha \beta}(k-1)+\frac{1-e^{-T_{s c} / \hat{\tau}}}{\hat{R}_{s}} \boldsymbol{u}_{s 2}^{r e f}(k-1) \\
-\frac{e^{j \omega_{r} T_{s c}-} e^{-T_{s c} / \hat{\tau}}}{\hat{R}_{s}+j \omega_{r} \hat{L}}\left(j \omega_{r} \boldsymbol{\psi}_{r}^{\alpha \beta}(k-1)+\boldsymbol{u}_{d}^{\alpha \beta}(k-1)\right)
\end{gathered}
$$

Considering the actual disturbance $\boldsymbol{u}_{d}^{\alpha \beta}$ is unknown, the applied voltage can be computed with the estimated disturbance as

$$
\begin{aligned}
& \boldsymbol{u}_{s}^{r e f}(k-1)=\boldsymbol{u}_{s 1}^{r e f}(k-1) \\
& \quad+\frac{\hat{R}_{s}}{1-e^{-T_{s c} / \tau}} \cdot \frac{e^{j \omega_{r} T_{s c}-} e^{-T_{s c} / \hat{\tau}}}{\hat{R}_{s}+j \omega_{r} \hat{L}} \hat{\boldsymbol{u}}_{d}^{\alpha \beta}(k-1)
\end{aligned}
$$

where $\boldsymbol{u}_{s 1}^{\text {ref }}$ was expressed in (9). When the above voltage is applied, the actual stator current at $(k)$ th instant can be obtained as

$$
\begin{gathered}
\boldsymbol{i}_{s}^{\alpha \beta}(k)=e^{-T_{s c} / \hat{\tau}} \boldsymbol{i}_{s}^{\alpha \beta}(k-1)+\frac{1-e^{-T_{s c} / \hat{\tau}}}{\hat{R}_{s}} \boldsymbol{u}_{s}^{r e f}(k-1) \\
-\frac{e^{j \omega_{r} T_{s c}-} e^{-T_{s c} / \hat{\tau}}}{\hat{R}_{s}+j \omega_{r} \hat{L}}\left(j \omega_{r} \boldsymbol{\psi}_{r}^{\alpha \beta}(k-1)+\boldsymbol{u}_{d}^{\alpha \beta}(k-1)\right)
\end{gathered}
$$

Considering (13), a sliding-mode disturbance adaptation scheme is developed as

$$
\begin{aligned}
\boldsymbol{e}_{i}(k) & =\boldsymbol{i}_{s}^{r e f}(k)-\boldsymbol{i}_{s}^{\alpha \beta}(k) \\
\hat{\boldsymbol{u}}_{d}^{\alpha \beta}(k) & =\hat{\boldsymbol{u}}_{d}^{\alpha \beta}(k-1) e^{j \omega_{r} T_{s c}}+\lambda Z\left(\boldsymbol{e}_{i}(k)\right)
\end{aligned}
$$

where $\lambda>0$ is adaptation gain of the disturbance and $Z(\bullet)$ is a boundary switching function which is defined as

$$
Z\left(\boldsymbol{e}_{i}\right)= \begin{cases}\frac{\boldsymbol{e}_{i}}{\left|\boldsymbol{e}_{i}\right|} & \text { if }\left|\boldsymbol{e}_{i}\right|>\sigma \\ \boldsymbol{e}_{i} & \text { if }\left|\boldsymbol{e}_{i}\right|<\sigma\end{cases}
$$

with $\sigma$ as the boundary width. Subtracting (17) from (15) yields

$$
\boldsymbol{e}_{i}(k)=\frac{e^{j \omega_{r} T_{s c}-} e^{-T_{s c} / \hat{\tau}}}{\hat{R}_{s}+j \omega_{r} \hat{L}} \boldsymbol{e}_{u}(k-1)
$$

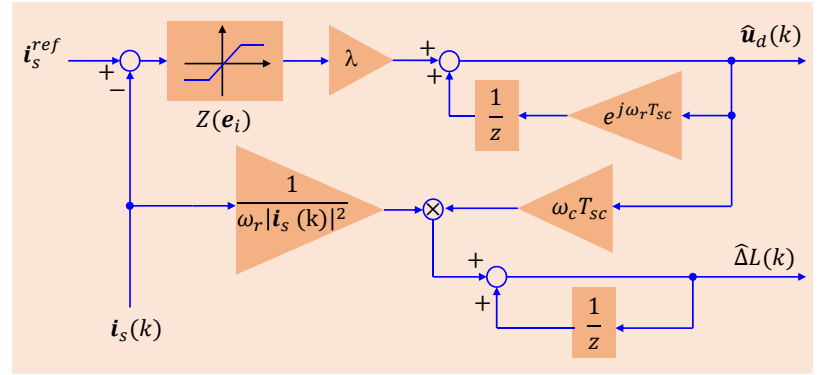

Fig. 2. Diagram of the proposed disturbance estimation with online inductance estimation.

where $\boldsymbol{e}_{u}(k-1)=\boldsymbol{u}_{d}^{\alpha \beta}(k-1)-\hat{\boldsymbol{u}}_{d}^{\alpha \beta}(k-1)$ is the estimation error of disturbance. If $\lambda$ is designed as

$$
\lambda=h e^{j \omega_{r} T_{s c}} \frac{\hat{R}_{s}+j \omega_{r} \hat{L}}{e^{j \omega_{r} T_{s c}-} e^{-T_{s c} / \hat{\tau}}}
$$

the relationship between $\hat{\boldsymbol{u}}_{d}^{\alpha \beta}$ and $\boldsymbol{u}_{d}^{\alpha \beta}$ can be calculated based on (18)-(22) as

$$
F(z)=\frac{\hat{\boldsymbol{u}}_{d}^{\alpha \beta}(z)}{\boldsymbol{u}_{d}^{\alpha \beta}(z)}=\frac{h e^{j \omega_{r} T_{s c}} z^{-1}}{1-(1-h) e^{j \omega_{r} T_{s c}} z^{-1}}
$$

To ensure stability of the disturbance estimation, a sufficient large $h$ should be employed according to classic sliding-mode control theory. Noted that $F(z)$ is derived when the state variable stays within boundary width, i.e., $\left|\boldsymbol{e}_{i}\right|<\sigma$. It is clear that $F\left(e^{j \omega_{r} T_{s c}}\right)=1$. Thus, the developed method can track the disturbance at fundamental frequency without magnitude error and phase delay, as also be confirmed by the bode plot in Fig. 1.

After obtaining the estimated disturbance $\hat{\boldsymbol{u}}_{d}^{\alpha \beta}(k)$, it is used in (14) instead of unknown $\boldsymbol{u}_{d}^{\alpha \beta}(k)$ to calculate voltage reference. It should be noted that in practical application, there is usually one-step delay between the calculated voltage and the applied voltage. To compensate for the delay, (14) should be shifted forward by one step. The required stator current $\boldsymbol{i}_{s}(k+1)$ and $\hat{\boldsymbol{u}}_{d}^{\alpha \beta}(k+1)$ can be simply predicted by (12) and shifting (19) one-step ahead respectively.

\section{B. Inductance Adaptation}

From (11), the disturbance voltage can be rewritten as (24) during steady state.

$\boldsymbol{u}_{d}^{\alpha \beta}(k)=-\Delta L \cdot j \omega_{r} \boldsymbol{i}_{s}^{\alpha \beta}(k)-\Delta R \boldsymbol{i}_{s}^{\alpha \beta}(k)-j \omega_{r} \cdot \Delta \psi e^{j \theta_{r}(k)}$

From (24), the following equation can be derived

$\boldsymbol{u}_{d}^{\alpha \beta}(k) \otimes \boldsymbol{i}_{s}^{\alpha \beta}(k)=-\Delta L \cdot \omega_{r}\left|i_{s}^{\alpha \beta}(k)\right|^{2}-\omega_{r} \Delta \psi e^{j \theta_{r}(k)} \otimes \boldsymbol{i}_{s}^{\alpha \beta}(k)$

where $\otimes$ denotes cross product of two complex vectors. Considering $i_{d}$ is controlled as zero, and $q$-axis is perpendicular to the rotor flux vector, $\Delta \psi e^{j \theta_{r}(k)} \otimes \boldsymbol{i}_{s}^{\alpha \beta}(k)=0$ holds. Hence, inductance estimation error can be derived as

$$
\Delta L=\frac{\boldsymbol{i}_{s}^{\alpha \beta}(k) \otimes \boldsymbol{u}_{d}^{\alpha \beta}(k)}{\omega_{r}\left|\boldsymbol{i}_{s}^{\alpha \beta}(k)\right|^{2}}
$$




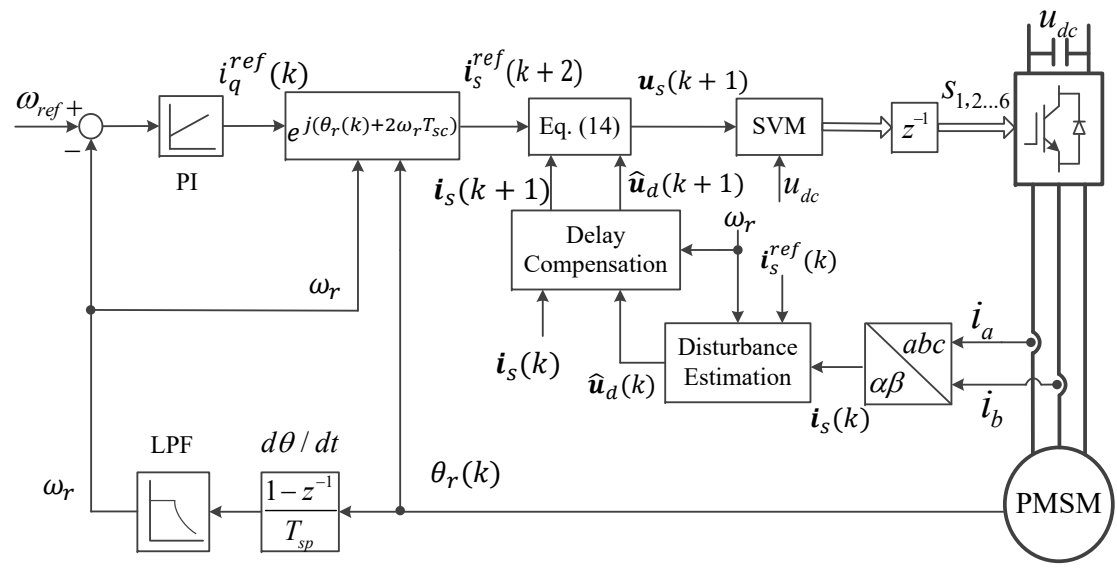

Fig. 3. Schematic diagram of the whole control system.

TABLE I

MACHINE AND CONTROL PARAMETERS

\begin{tabular}{lcc}
\hline Number of pole pairs & $N_{p}$ & 3 \\
Stator resistance & $R_{s}$ & $1.75 \Omega$ \\
Inductance & $L$ & $14.78 \mathrm{mH}$ \\
Rotor flux & $\psi_{f}$ & $0.1045 \mathrm{~Wb}$ \\
Control period & $T_{s c}$ & $2 \mathrm{~ms}$ \\
Parameter 1 & $\sigma$ & 0.1 \\
Parameter 2 & $h$ & 0.25 \\
Parameter 3 & $\omega_{c}$ & 10 \\
\hline
\end{tabular}

Based on (26), the inductance error is estimated as

$$
\Delta \hat{L}(k)=\Delta \hat{L}(k-1)+\omega_{c} T_{s c} \frac{\boldsymbol{i}_{s}^{\alpha \beta}(k) \otimes \hat{\boldsymbol{u}}_{d}^{\alpha \beta}(k)}{\omega_{r}\left|\boldsymbol{i}_{s}^{\alpha \beta}(k)\right|^{2}}
$$

With the estimated inductance error $\Delta \hat{L}(k)$, the corrected inductance $\hat{L}(k)=\hat{L}+\Delta \hat{L}(k)$ is used in the control algorithm to further improve the transient performance. The diagram of the proposed disturbance estimation with inductance estimation is shown in Fig. 2.

\section{Simulation and Experimental Results}

To verify effectiveness of the proposed method, simulation and experimental tests were carried out on a two-level inverter fed PMSM drive. For comparison, prior method as shown in [8] was also performed. The motor and control parameters summarized in Table I are used in both simulation and experimental tests. The control diagram of the proposed method was shown in Fig. 3. The rotor speed is regulated by a PI controller which gives $q$-axis current reference $i_{q}^{r e f}$ and $d$-axis current reference $i_{d}^{r e f}$ is set as zero in this paper.

Fig. 4 shows dynamic performance of the proposed method and the prior method when the speed reference steps from 50 $\mathrm{Hz}$ to $100 \mathrm{~Hz}$. Since the switching frequency is only $500 \mathrm{~Hz}$, pulse ratio would finally decreases to 5 in this test. However, both methods are stable with good tracking performance. In the proposed method, $d$-axis current $i_{d}$ is well kept at zero without influenced by the fast change of $i_{q}$, indicating
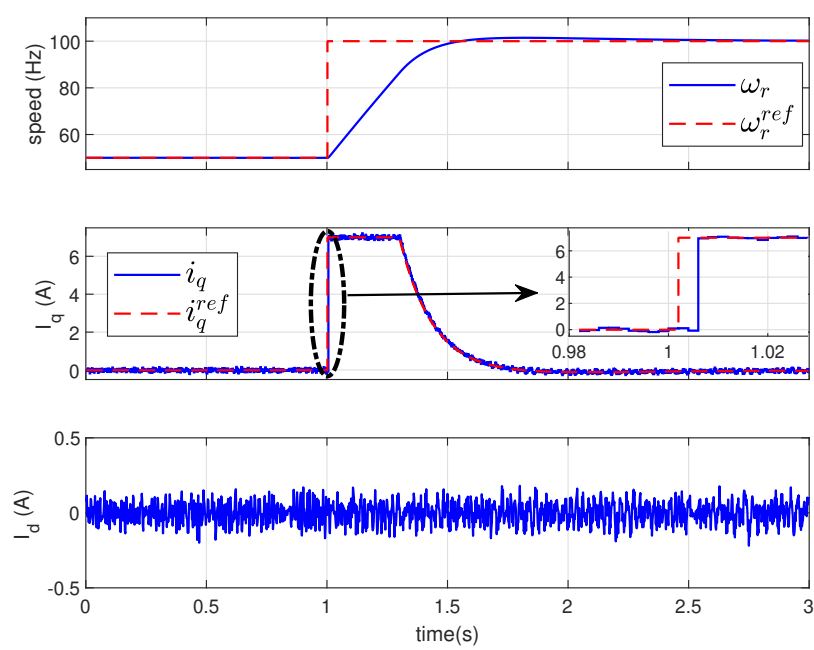

(a)
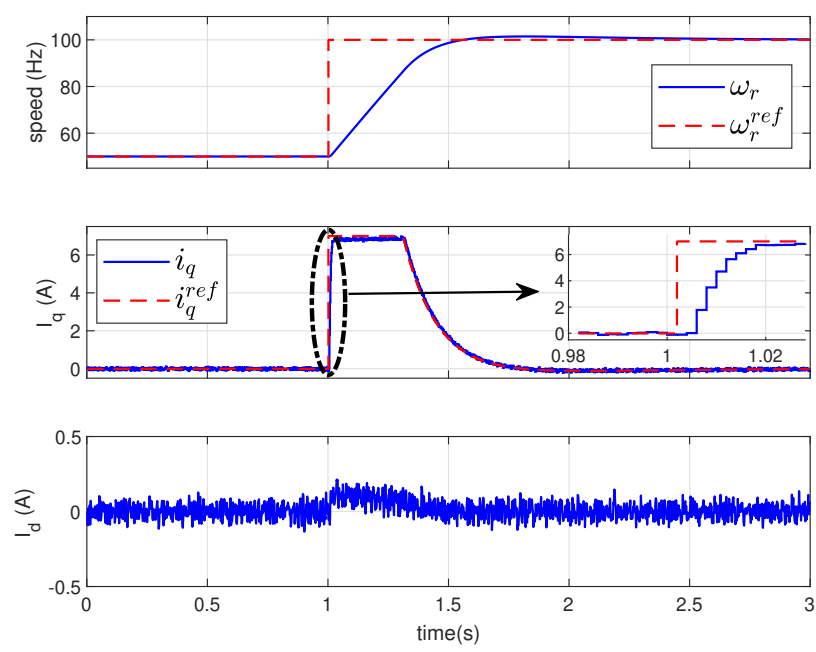

(b)

Fig. 4. Simulation results of (a) the proposed method and (b) the prior method with accurate motor parameters $\left(\omega_{r}\right.$ increases from $50 \mathrm{~Hz}$ to $100 \mathrm{~Hz}$ ).

decoupled control of $i_{d}$ and $i_{q}$ is well achieved. It is clear that 

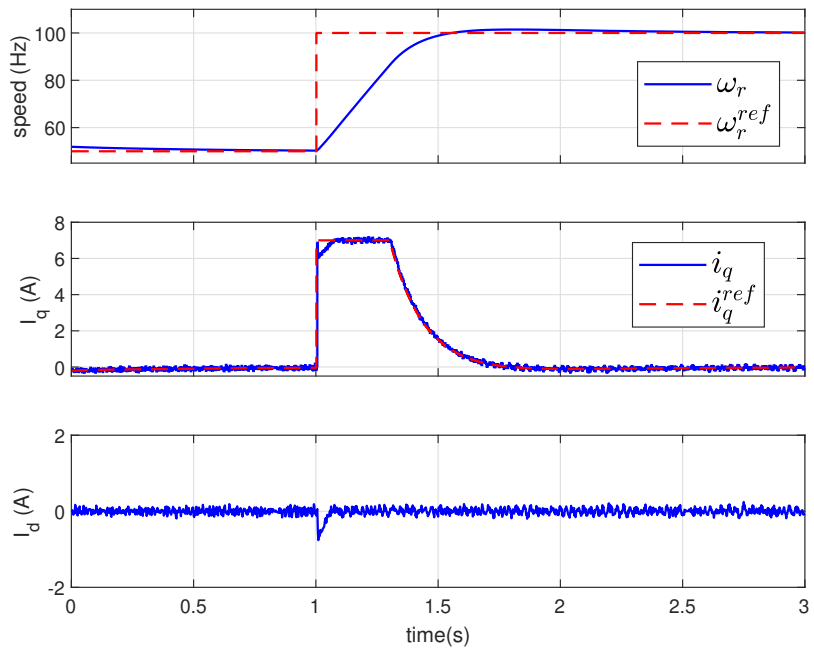

(a)
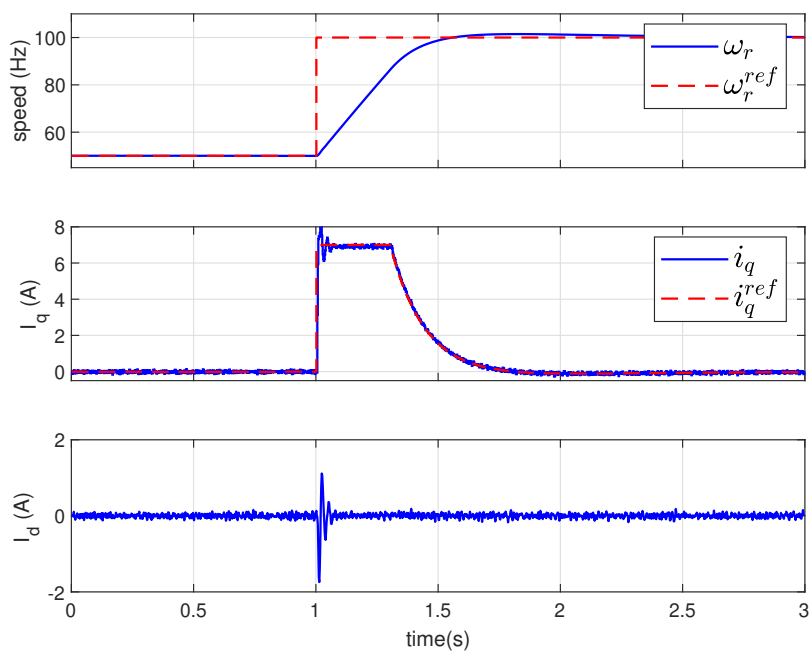

(b)

Fig. 5. Simulation results of (a) the proposed method and (b) the prior method with mismatched motor parameters $\left(\hat{L}=2 L, \hat{R}=0.5 R\right.$ and $\left.\hat{\psi}_{r}=0.5 \psi_{r}\right)$.

the proposed method shows shorter settling time and slightly better decoupling performance when compared with the prior method.

Fig. 5 shows dynamic responses when inaccurate motor parameters, i.e., $\hat{L}=2 L, \hat{R}=0.5 R$ and $\hat{\psi}_{r}=0.5 \psi_{r}$, were used in the control algorithm. Apparently, the prior discrete-time controller shows deteriorated performance with parameter mismatches. Significant oscillations and obvious coupling between $i_{d}$ and $i_{q}$ can be seen during such transient process. By comparison, one can see that the proposed method performs better under the same test conditions.

Fig. 6 shows the estimated inductance with different initial errors. It can be seen that with the proposed method, the estimated inductance can smoothly converge to its actual value. Thus, inductance error has no influence on the proposed DPCC if online inductance estimation is enabled.

Fig. 7 shows responses of the proposed method when the motor accelerates from standstill to $100 \mathrm{~Hz}$ with only $300 \mathrm{~Hz}$

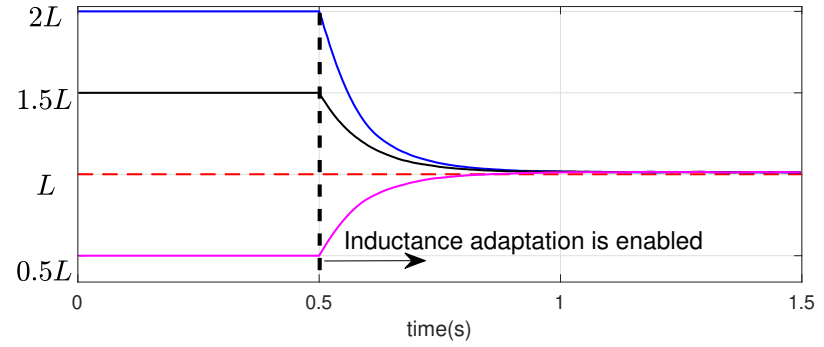

Fig. 6. Simulation results of the estimated inductance $\hat{L}$ with initial errors as $L, 0.5 L$ and $-0.5 L$ respectively.
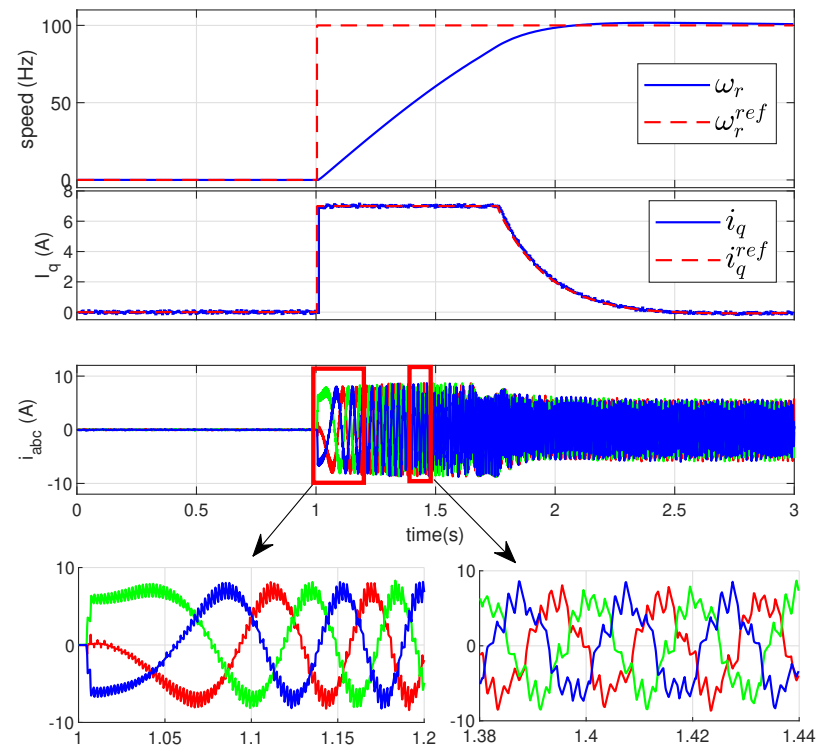

Fig. 7. Simulated responses of starting the motor from standstill to $100 \mathrm{~Hz}$ with $250 \mathrm{~Hz}$ switching frequency.

switching frequency. Due to very low switching frequency, the stator current is somewhat distorted with large harmonics. However, the system is still stable even when the pulse ratio is only 2.5. This test confirms that the proposed method can work effectively and stably even under extremely low pulse ratios, which is very suitable for high power or high speed motor drives.

Fig. 8 shows experimental results of the proposed method and the prior method when the estimated inductance is half of its actual value. It is clearly seen that both methods can stably operate when the pulse ratio decreases to 5 with mismatched motor parameters. However, the proposed method shows much better transient response than the prior method in terms of smaller settling time and better decoupled control performance.

Fig. 9 shows experimental results of the proposed method when the motor is running at $100 \mathrm{~Hz}$ with half load. It is clearly seen that there are only 5 samples for both $i_{d}$ and $i_{q}$ per one fundamental period. Although stator current is distorted due to low switching frequency, the system operates stably. 


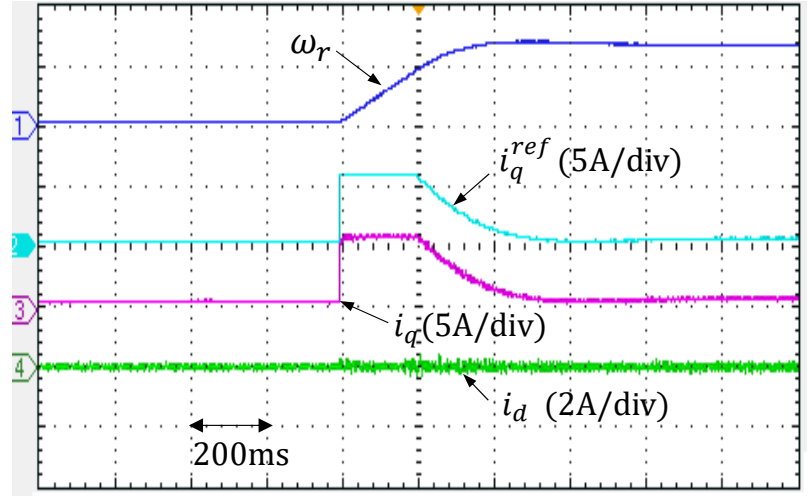

(a)

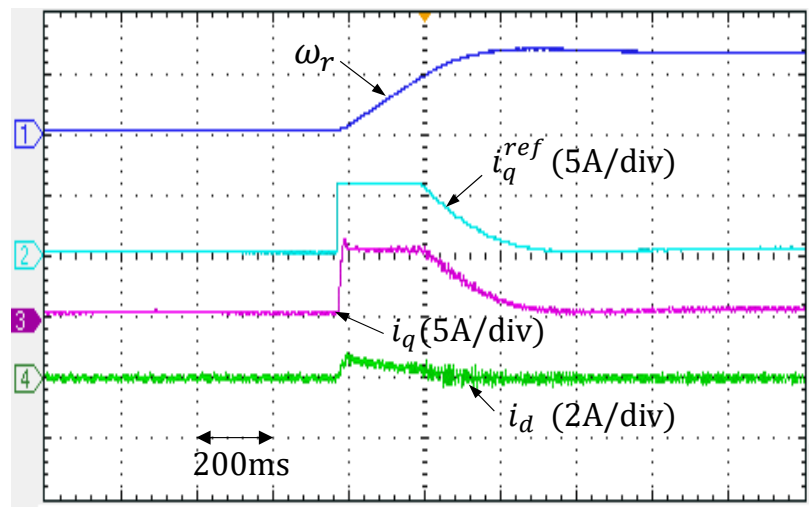

(b)

Fig. 8. Experimental results of (a) the proposed method and (b) the prior method with $500 \mathrm{~Hz}$ switching frequency and $\hat{L}=0.5 L$ ( $\omega_{r}$ increases from $5 \mathrm{~Hz}$ to $100 \mathrm{~Hz}$ ).

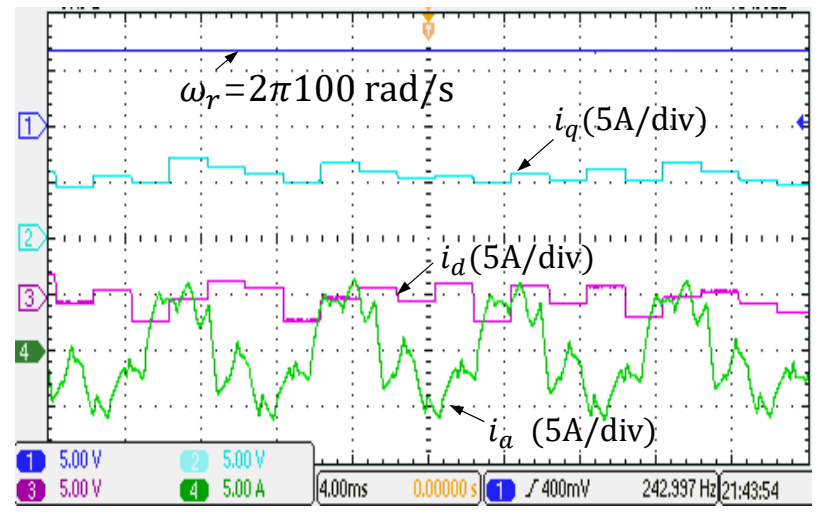

Fig. 9. Experimental results of the proposed method with $500 \mathrm{~Hz}$ switching frequency and half load. $\left(\omega_{r}=2 \pi 100 \mathrm{rad} / \mathrm{s}\right)$.

\section{CONCLUSION}

In this paper, an robust digital current control scheme was designed and verified for PMSM drive with low switching frequency. It was shown that prior digital current controller suffers significantly deteriorated control performance during transient process when inaccurate motor parameters are used in the controller. With the proposed inductance adaptation scheme, the mismatched inductance can be accurately corrected. Hence, the side-impact of inductance error can be eliminated. Although transient performance slightly degrades when there are resistance and rotor flux errors, it still performs better than the prior method. When the pulse ratio is decreased below 3 , the proposed method can still work stably. In conclusion, the proposed digital current controller can work well under low pulse ratio and it is robust against parameter mismatches. Simulation and experimental tests validated the effectiveness of the proposed method.

\section{REFERENCES}

[1] Y. Wang, S. Tobayashi, and R. D. Lorenz, "A low-switching-frequency flux observer and torque model of deadbeat direct torque and flux control on induction machine drives," IEEE Trans. Ind. Appl., vol. 51, no. 3, pp. 2255-2267, May 2015.

[2] H. Yang, Y. Zhang, J. Liang, B. Xia, P. D. Walker, and N. Zhang, "Deadbeat control based on a multipurpose disturbance observer for permanent magnet synchronous motors," IET Electr. Power Appl., vol. 12, no. 5, pp. 708-716, 2018.

[3] W. Wang, J. Zhang, and M. Cheng, "A dual-level hysteresis current control for one five-leg vsi to control two pmsms," IEEE Trans. Power Electron., vol. 32, no. 1, pp. 804-814, Jan 2017.

[4] J. Lara, J. Xu, and A. Chandra, "A novel algorithm based on polynomial approximations for an efficient error compensation of magnetic analog encoders in pmsms for evs," IEEE Trans. Ind. Electron., vol. 63, no. 6, pp. 3377-3388, June 2016.

[5] S. C. Yang and G. R. Chen, "High-speed position-sensorless drive of permanent-magnet machine using discrete-time emf estimation," IEEE Trans. Ind. Electron., vol. 64, no. 6, pp. 4444-4453, June 2017.

[6] H. Yang, Y. Zhang, G. Yuan, P. D. Walker, and N. Zhang, "Hybrid synchronized pwm schemes for closed-loop current control of highpower motor drives," IEEE Trans. Ind. Electron., vol. 64, no. 9, pp. 6920-6929, Sept 2017.

[7] B.-H. Bae and S.-K. Sul, "A compensation method for time delay of full-digital synchronous frame current regulator of PWM AC drives," IEEE Trans. Ind. Appl., vol. 39, no. 3, pp. 802-810, 2003.

[8] H. Kim, M. Degner, J. Guerrero, F. Briz, and R. Lorenz, "Discrete-time current regulator design for ac machine drives," IEEE Trans. Ind. Appl., vol. 46, no. 4, pp. $1425-1435$, july-aug. 2010.

[9] J. Holtz, J. Quan, J. Pontt, J. Rodriguez, P. Newman, and H. Miranda, "Design of fast and robust current regulators for high-power drives based on complex state variables," IEEE Trans. Ind. Appl., vol. 40, no. 5, pp. 1388 - 1397, sept.-oct. 2004.

[10] N. Hoffmann, F. W. Fuchs, M. P. Kazmierkowski, and D. Schröder, "Digital current control in a rotating reference frame - part i: System modeling and the discrete time-domain current controller with improved decoupling capabilities," IEEE Trans. Power Electron., vol. 31, no. 7, pp. 5290-5305, July 2016.

[11] S. C. Yang and G. R. Chen, "High speed position sensorless drive of permanent magnet machine using discrete-time emf estimation," IEEE Trans. Ind. Electron., vol. 64, no. 6, pp. 4444 - 4453, 2017. 